\title{
ESTIMATION OF ROTATION PARAMETERS OF THREE-DIMENSIONAL IMAGES BY SPHERICAL HARMONICS ANALYSIS
}

\author{
Alexey Rozhentsov*, Irina Egoshina, Alexey Baev, Daniil Chernishov \\ Volga State University of Technology, Yoshkar-Ola, Russian Federation
}

The article describes a method for estimating the rotational parameters of three-dimensional objects defined as a cloud of points in three-dimensional space, which is less complex compared to other methods and it can ensure a single-valued solution. The authors propose an approach of vector-field modelsto parametrize images of complex three-dimensional objects. The paper discusses the ways for calculating the expansion coefficients in the basis of spherical harmonics for images of three-dimensional pointcloud objects. The authors offer an approach that provides the possibility of estimating the rotation parameters of three-dimensional objects from the values of the expansion coefficients in the basis of spherical harmonics.

Key words: Rotation of three-dimensional objects; Pointcloud; Estimation of parameters; Spherical harmonics

\section{INTRODUCTION}

Estimation of rotation parameters of a three-dimensional object is an objective for processing images [1-7], 3D modelingand remodeling of an object [8-13].

There are a number of approaches to solve the problem. The first approach is based on the preliminary identification of marks within the structure of the reference and observable three-dimensional objects. Thus, if we have at least three identified marks, we can calculate the rotation matrix.This approach can beappliedin astro-orientation systems [14], insystems for highlightobjectrecognition in radar images by means of iterative angular matching of rotation parameters of 3D objects in context of a priori uncertainty of the angular parameters [15-17], and in order to process images of polyhedron [18].

In the case when the identification of marks is not feasible, or it has been executed with errors, the problem of estimating rotation parameters on the basis of this approach is either not solved, or solved with errors.

The second approach is based on the use of correlation algorithms for image processing of three-dimensional objects [19], but it is rathercomplex in context ofa priori uncertainty regarding the transformation parameters of scaling, rotating, and transferring. The Hoch-based transformation approaches[20-24] are of lower complexity, however, the requirements for computational resources are quite high, and consequently, these approaches have less noise immunity when compared to the correlation algorithms.

The third approach is based on the use of functional descriptions of three-dimensional surfaces that do not require knowledge of the numbering of samples of a three-dimensional object. The paper [25], gives a solution to the problem of estimating rotation parameters on the basis of an analysis of the projecting functions of a quaternion variable. One of the drawbacks of this approach is that it is limited to be applicable forstellar shapes only and it has less noise immunity when compared to the correlation algorithms. The papers[26, 27], resent a technique for estimating rotational parameters on the basis of an analysis of spectral coefficients for the expansion of a function describing the surface of an object in the basis of spherical harmonics. The downside of the technique is the use of iterative procedures to determine Euler angles, which complicates the procedure and does not guarantee the uniqueness of solution.

Thus, the objective of the work is to develop an analytical method for estimating rotation parameters of three-dimensional point cloudobjects in three-dimensional space, which is of low complexity and provides a single-valued solution.

\section{THEORY AND EXPERIMENTAL METHODS USED}

\section{Parameterization of images of three-dimensional objects}

While trying to solve problems of processing volumetric images we often use methods based on a functional description of the surface of a three-dimensional object [25-29]. For this purpose, the references that define the surface of the object should be parametrized, i.e. they should be uniquely defined in the coordinate system used to represent the surface using a particular system of functions. When spherical harmonics or methods based on the projection of references onto a single sphere are applied, all references of the surface of the object should be given in a spherical coordinate system. Parameterization for stellar shapes can be performed routinely by constructing vectors from any point inside the figure, for example, from the center of gravity, to the points on the surface of the object. However, this method is not applicable for figures of a more complex shape, because in such a case, the ambiguity of setting the references of 

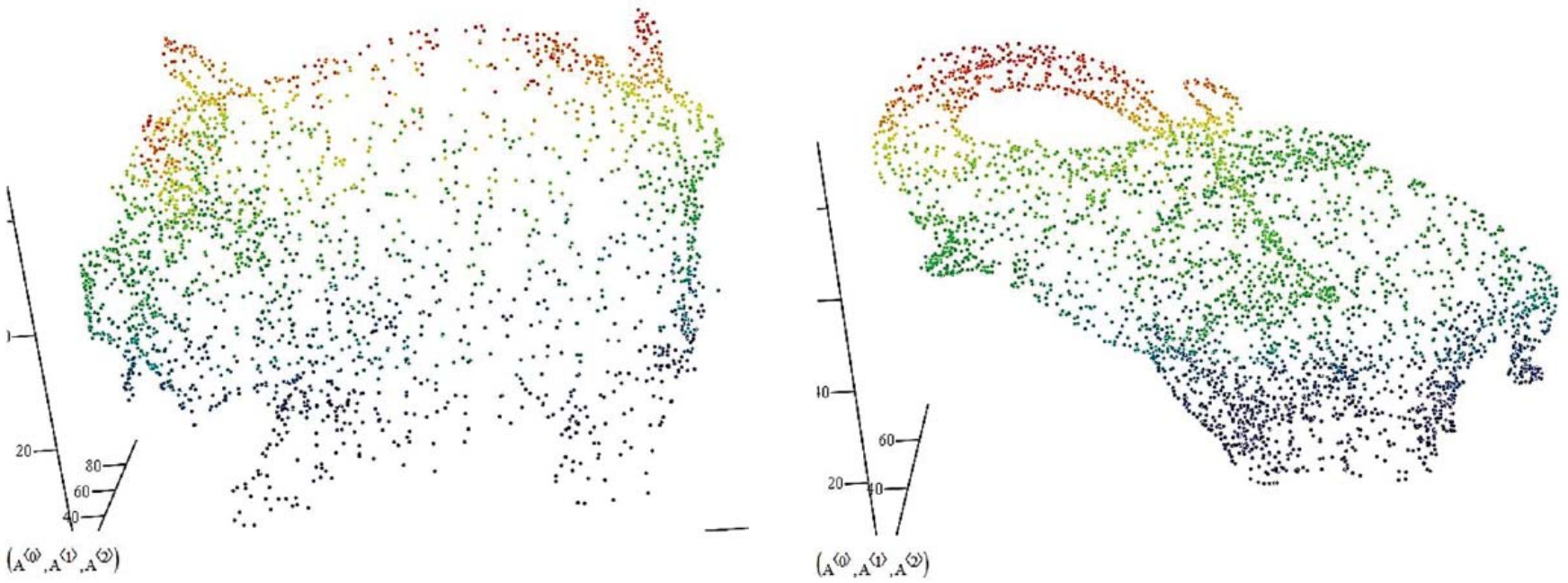

Figure 1: Point clouds that define the surfaces of the test objects

the object arises.

The paper [30] describes the methods of parametrization, which is often used for processing real images. The drawbacks of the methods are the dependence of the results of parametrization on the choice of initial conditions, and the need for approximation of surface fragments when constructing latitude and longitude lines. Additional optimization of the obtained parameterization result is also required.

In this paper, we offer a vector-field model approach. Let us consider a scene with a spatial object whose surface is given by a point cloud obtained, for example, as a result of scanning the object (Fig. 1).

We associate with each $n$-th point of the surface a certain charge, the coordinates of which are given by a $\mathrm{q}(n)$ three-dimensional vector. Let the coordinates of some point of the scene be given by a three-dimensional vector. Then the vector of electric field intensity created by all charges at this point is determined by the formula 1 .

Each charge can be assigned with the electric field lines radiating from this charge and subjected to deformation in space due to the influence of the field of other charges. Let the beginning of the force line coincide with the position of the charge in the scene, i.e. $\mathrm{v}_{0}(n)=\mathrm{q}(n)$, and the initial direction coincide with the direction of the normal vector to the surface at a given point. To determine the position in space of the next point of the force line, it is essential to determine the direction of the electric field vector at a given point and to move in the direction of this vector by some value $\varepsilon$. Then the coordinate of the point of the force line in the next step is determined by the formula 2 . The process of force line designing continues until it extends beyond the sphere of a given radius. The angular coordinates of the intersection point of the sphere by the force line are taken as the result of the parametrization of the $n$-th point. Figure 2 shows the results of designing the force lines of the electric field for the test objects shown in figure1.

After each point of the surface is mapped onto a sphere, one can perform its expansion in an orthogonal basis, for example, in the basis of spherical harmonics by 3 .

where $P_{I}^{m}(\cos \theta)$ - aretheassociated Legendre polynomials of degree land order $m$ [26, 27, 30]. The calculation of the expansion coefficients in the basis of spherical harmonics should be done by the formula 4 .

i.e. the surface should be represented as a function in spherical coordinates. In practical terms a functional description of the surface may be absent; i.e. initial data for processing three-dimensional surfaces are usually point clouds obtained as a result of scanning. In this case, calculations in accordance with (4) are impossible and the expansion coefficients are determined by solving a

$$
\begin{gathered}
\mathbf{e}(\mathbf{p})=\sum_{n=0}^{N-1} \frac{(\mathbf{p}-\mathbf{q}(n))}{|\mathbf{p}-\mathbf{q}(n)|^{3}} \quad(1) \quad \mathbf{v}_{m+1}(n)=\mathbf{v}_{m}(n)+\frac{\mathbf{e}\left(\mathbf{v}_{m}(n)\right)}{\left|\mathbf{e}\left(\mathbf{v}_{m}(n)\right)\right|} \\
Y_{m}^{l}(\theta, \phi)=\frac{1}{2} \sqrt{\frac{(2 l+1)}{2 \pi} \frac{(l-|m|) !}{(l+|m|) !}} \cdot P_{l}^{|m|}(\cos \theta) \cdot e^{i m \phi}, l=0,1,2, \ldots ; m=0, \pm 1, \pm 2, \ldots, \pm l \\
\rho_{l, m}=\int_{0}^{2 \pi} \int_{0}^{\pi} f(\theta, \phi) Y_{m}^{l}(\theta, \phi) d \theta d \phi, l=0,1,2, \ldots ; m=0, \pm 1, \pm 2, \ldots, \pm l
\end{gathered}
$$



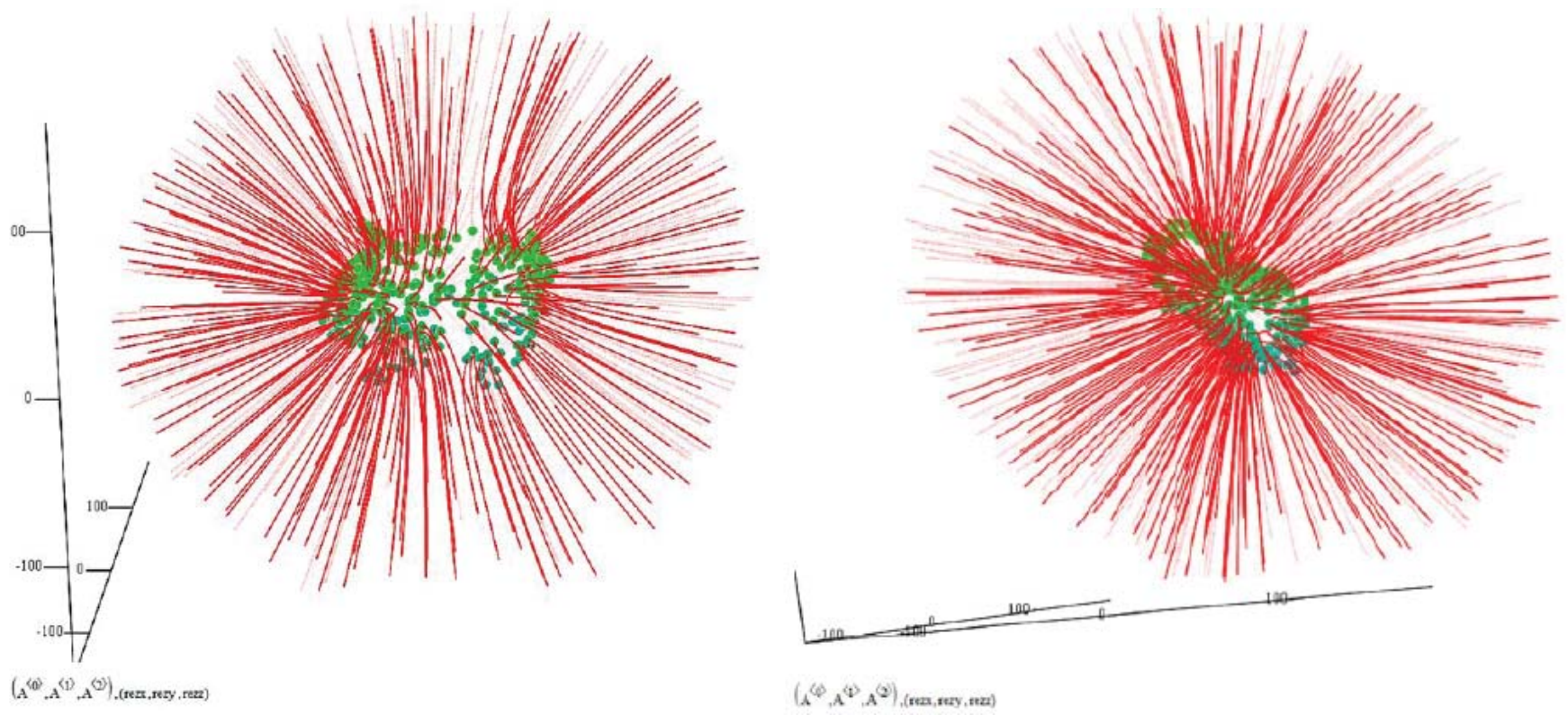

Figure 2: Example of designing the electric field lines for 3D surface parametrization

system of linear equations by:

$$
\sum_{l=0}^{L-1} \sum_{m=-l}^{l} Y_{m}^{l}(\theta(n), \phi(n)) \rho_{l, m}=f(\theta(n), \phi(n)), n=0,1, \ldots, N-1
$$

or in the matrix form:

$$
\mathrm{YP}=\mathrm{F} \text {, }
$$

where $N$ is the number of references that define a three-dimensional surface. The parameter $L$ is set, on the one hand, on the basis of the required accuracy of the representation of the object in the basis of spherical harmonics, on the other hand, based on the possibility of a correct solution of the system (5). In such a case:

$$
N \geq L^{2}
$$

If $N>L^{2} N>L^{2}$ i.e. when the number of equations exceeds the number of unknowns, the solution of the system can be found by the method of least squares [31]:

$$
\mathrm{P}=\left(\left(\mathrm{Y}^{\mathrm{t}}\right)^{*} \mathrm{Y}\right)\left(\left(\mathrm{Y}^{\mathrm{t}}\right)^{*} \mathrm{~F}\right) \text {. }
$$

In the case of three-dimensional parametrized surfaces, it is essential to separately perform the expansion in coordinates $x, y, z$, i.e. three vectors of coordinate values are formed by:

$$
\begin{gathered}
f x(\theta(n), \phi(n))=x(n), \\
f x(\theta(n), \phi(n))=y(n), \\
f x((n), \phi(n))=z(n), \\
n=0,1, \ldots, N-1 .
\end{gathered}
$$

Then the expansion coefficients in a spherical basis for functions with respect to the corresponding coordinates can be determined by:

$P_{x}=\left(\left(Y^{\top}=Y\right)-1\left(\left(Y^{\top}\right)^{*} F_{x}\right)\right.$,

$P_{y}=\left(\left(Y^{\top}=Y\right)-1\left(\left(Y^{\top}\right)^{\star} F_{y}\right)\right.$,

$P_{\mathrm{z}}=\left(\left(\mathrm{Y}^{\top}=\mathrm{Y}\right)-1\left(\left(\mathrm{Y}^{\top}\right)^{*} \mathrm{~F}_{\mathrm{z}}\right)\right.$,

where $F_{x^{\prime}}, F_{y^{\prime}}, F_{z}$ are the vectors containing references that specify the coordinates of points on the surface of the object.

\section{EXPERIMENTAL SECTION}

Figure 3 shows the results of approximation of the objects presented in figure 1 , as well as the results of approximation of the test objects of geometric primitives: a cube, and a cone.If $L=11$ and above we can reach a good quality of approximation of three-dimensional surfaces when using the proposed method for their parametrization. Figure 4 shows the comparative results of the approximation of the test object, described in the paper [30]. The results show that the method proposed provides a comparable quality of surface approximation.

\section{ESTIMATION OF ROTATION PARAMETERS OF 3-D IMAGES}

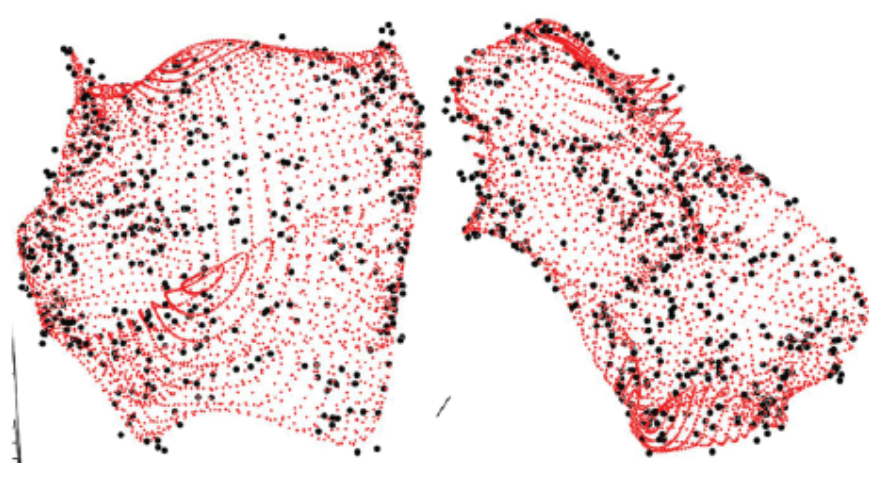

a)

Figure 3: Examples of approximation ofthe test objects: a) approximation of the objects presented in Figure 1 


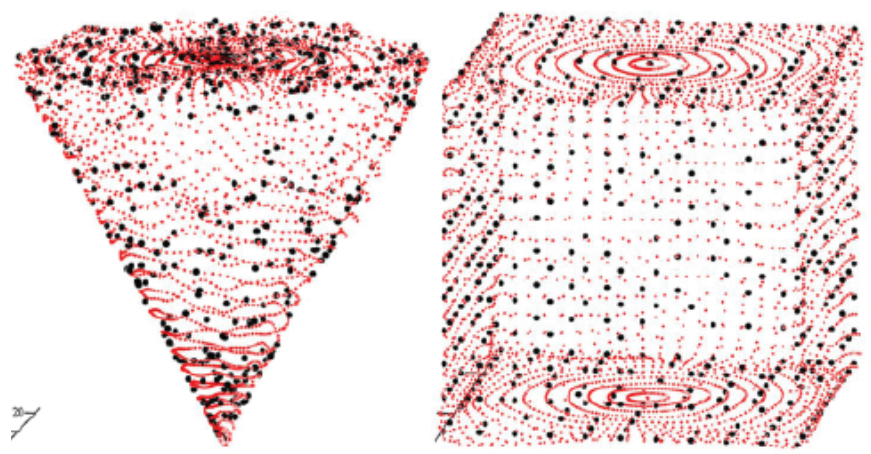

b)

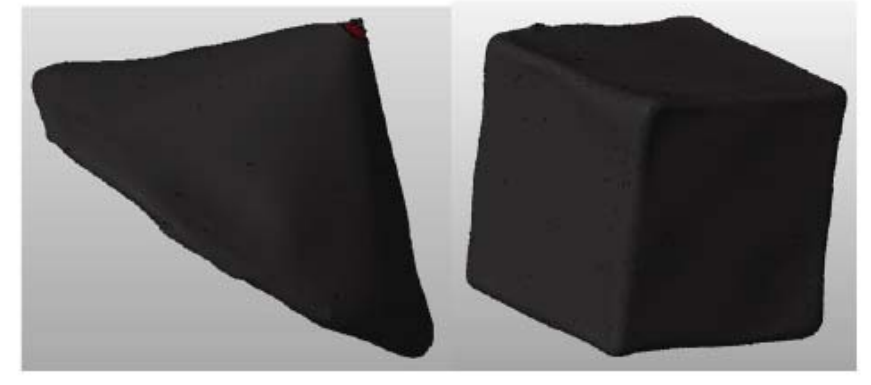

c)

Figure 3: Examples of approximation ofthe test objects:

b) approximation of thetest objects defining a cone and a cube

c) an example of designing a surface from approximated points for a cone and a cube

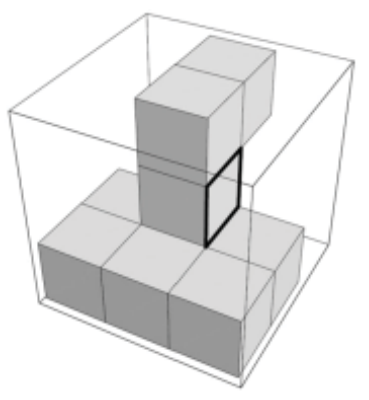

a)

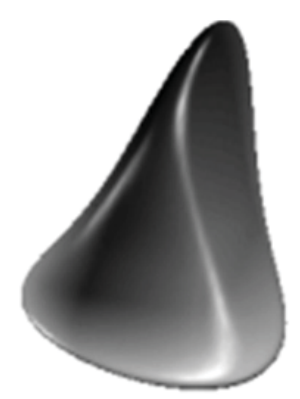

b)

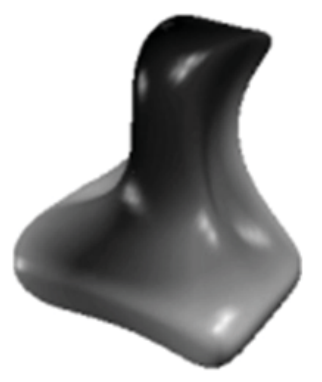

c)

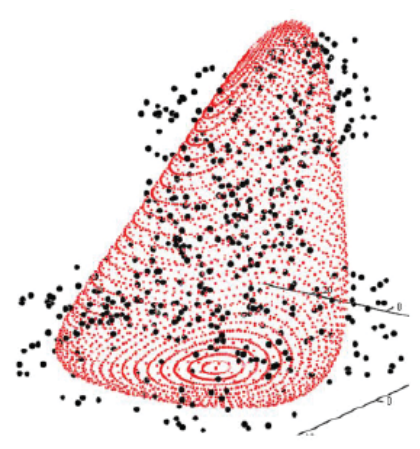

d)

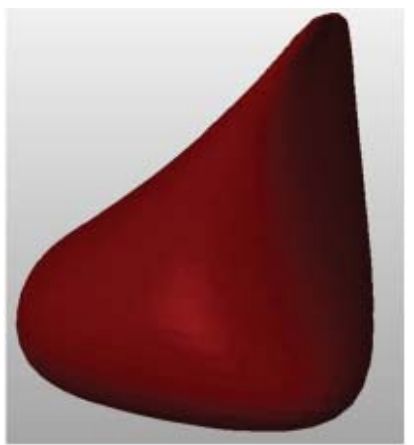

f)

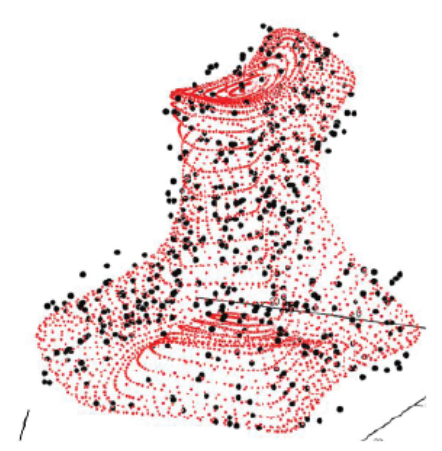

e)

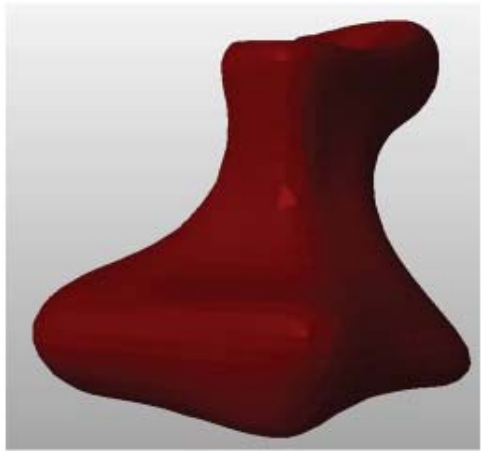

g)

Figure 4: Comparison of the results of approximation, a) test three-dimensional object [16]; b), c) the results of designing the approximated surface, respectively, when $L=1$ and $L=2$ [16];d), e) a pointcloud and the results of approximation using the proposed parametrization method when $L=1$ and $L=2 ; f$ )

g) the result of the surface visualization 
If a three-dimensional image has been subjected to a rotation transformation, then its restoration based on the obtained expansion coefficients in the basis of spherical harmonics will be as follows:

where $\rho_{I m}{ }^{(r)}$ is the expansion coefficients of the rotated object. A number of papers, for example, [26, 27] show

$$
f^{(r)}(\theta(n), \phi(n))=\sum_{l=0}^{L-1} \sum_{m=-l}^{l} Y_{m}^{l}(\theta(n), \phi(n)) \rho_{l, m}^{(r)}
$$

that there is a relationship between the expansion coefficients of the reference and the rotated objects, given by the Wigner D-matrix:

$$
\rho_{l, m}^{(r)}=\sum_{n=-l}^{l} D_{n m}^{(l)}(\alpha, \beta, \gamma) \rho_{l, n}
$$

where

$$
\begin{aligned}
& \mathbf{D}_{n m}^{(l)}(\alpha, \beta, \gamma)= \\
& \sum_{\mu=\max (0, m-n)}^{\min (l+m, l-n)}(-1)^{\mu} \frac{\sqrt{(l+m) !(l-m) !(l+n) !(l-n) !}}{(l+m-\mu) ! \mu !(l-n-\mu) !(n-m+\mu) !} \times \\
& \times e^{-i(n \alpha+m \gamma)} \cdot \cos ^{2 i+m-n-2 \mu}\left(\frac{\beta}{2}\right) \cdot \sin ^{n-m+2 \mu}\left(\frac{\beta}{2}\right),
\end{aligned}
$$

where $l=0,1,2,3, \ldots, n, m=-I, \ldots, 0 \ldots+l, \alpha, \beta, \gamma$ - are Euler angles.

For example, if the Wigner D-matrix is:

$$
\begin{aligned}
& \mathbf{D}^{(1)}(\alpha, \beta, \gamma)= \\
& {\left[\begin{array}{ccc}
e^{-i \alpha} \frac{1+\cos \beta}{2} e^{-i \gamma} & -e^{-i \alpha} \frac{\sin \beta}{\sqrt{2}} & e^{-i \alpha} \frac{1-\cos \beta}{2} e^{i \gamma} \\
\frac{\sin \beta}{\sqrt{2}} e^{-t \gamma} & \cos \beta & -\frac{\sin \beta}{\sqrt{2}} e^{i \gamma} \\
e^{i \alpha} \frac{1-\cos \beta}{2} e^{-t \gamma} & e^{i \alpha} \frac{\sin \beta}{\sqrt{2}} & e^{i \alpha} \frac{1+\cos \beta}{2} e^{t \gamma}
\end{array}\right]}
\end{aligned}
$$

Thus, in order to estimate the rotation parameters of a three-dimensional object by analyzing the expansion coefficients in the basis of spherical harmonics, it is essential to find the elements of the matrices $D_{n m}{ }^{(1)}$. Let us consider the solution when $l=1$.

We write expression (8) in the matrix form:

$$
\rho_{1}^{(r)}=D^{(1)} \rho_{1}
$$

If only one set of coefficients and is used than the matrix elements can not be directly expressed from (11). It is necessary to have at least three sets of corresponding coefficients, which can be obtained in different ways. The first way is to allocate at least three objects in the scene and calculate the $\rho_{1}$ and $\rho_{1}{ }^{(r)}$ expansion coefficients for

$$
\begin{aligned}
\mathbf{P}_{1} & =\left[\begin{array}{lll}
\boldsymbol{\rho}_{1 \ni 1} & \boldsymbol{\rho}_{1 \ni 2} & \boldsymbol{\rho}_{1 \ni}
\end{array}\right], \\
\mathbf{P}_{1}^{(r)}= & {\left[\begin{array}{lll}
\boldsymbol{\rho}_{1}^{(r)} & \boldsymbol{\rho}_{1}^{(r)} & \boldsymbol{\rho}_{1}^{(r)}{ }_{\ni 3}
\end{array}\right] }
\end{aligned}
$$

each object. The combination of coefficients in $3 \times 3$ matrix allows us to express matrix from (11):

$$
\mathrm{D}^{(1)}=\mathrm{P}_{1}{ }_{1}^{(\mathrm{r})} \mathrm{P}_{1}^{-1}
$$

The main drawback of this method is the need to use the expansion coefficients of not less than three objects, which requires their preliminary selection and recognition in the scene.

To ensure the possibility of solving the problem of estimating parameters from one object, we propose the second method, based on the allocation of additional parameters of vectors $q(n)$, drawn from the center of gravity of the object to the references on its surface. Such aparameter can be the angle between the vector $q(n)$ and the normal vectorata given point on the surface of the object $n(n)$. The cosine of this angle can be defined as a standardized scalar product between the vectors $\mathrm{q}(n)$ and $\mathrm{n}(n)$ :

$$
\cos \phi(n)=\frac{(\mathbf{q}(n), \mathbf{n}(n))}{|\mathbf{q}(n)||\mathbf{n}(n)|}
$$

When we have values of the references of the vectors and the corresponding corners, we can form three 'quasi-figures' corresponding to the values $|\mathrm{q}(\theta, \phi)|,\left|\mathrm{q}_{\mathrm{Re}}(\theta, \phi)\right|=$ $|\mathrm{q}(\theta, \phi)| \cos \psi(n),\left|\mathrm{q}_{\mathrm{Re}}(\theta, \phi)\right|=\left|\mathrm{q}_{\mathrm{Im}}(\theta, \phi)\right| \sin \psi(n)$ andcalculate three vectors of the expansion coefficients:

$$
\begin{gathered}
\mathbf{P}_{1}=\left[\begin{array}{lll}
\boldsymbol{\rho}_{1|\mathbf{q}|} & \boldsymbol{\rho}_{1\left|\mathbf{q}_{\mathbf{R e}}\right|} & \boldsymbol{\rho}_{1\left|\mathbf{q}_{\mathrm{Im}}\right|}
\end{array}\right] \\
\mathbf{P}_{1}^{(r)}=\left[\begin{array}{lll}
\boldsymbol{\rho}_{1}^{(r)} & \left.\boldsymbol{\rho}_{1}^{(r)}\right|_{\left|\mathbf{q}_{\mathbf{R e}}\right|} & \left.\boldsymbol{\rho}_{1}^{(r)}\right|_{\mathbf{q}_{\mathrm{Im}} \mid}
\end{array}\right]
\end{gathered}
$$

Substituting the values $\mathrm{P}_{1}$ and $\mathrm{P}_{1}{ }^{(r)}$ obtained in accordance with (13) into (11), we can determine the matrix $D^{(1)}$.

\section{RESULTS}

Figure 5 shows images of the reference and rotated objects when $\alpha=130^{\circ}, \beta=35^{\circ}, \gamma=75^{\circ}$.

The following values $\mathrm{P}_{1}$ and $\mathrm{P}_{1}{ }^{(r)}$ were obtained for these objects:

$$
\begin{aligned}
& \mathbf{P}_{1}=\left[\begin{array}{ccc}
1,60-2,66 i & 0,61-0,63 i & 0,97-2,34 i \\
0,13 & 4,13 & -2,50 \\
-1,60-2,66 i & -0,61-0,63 i & -0,97-2,34 i
\end{array}\right] \\
& \mathbf{P}_{1}^{(r)}=\left[\begin{array}{ccc}
-2,19+1,28 i & 0,35-1,07 i & -2,23+2,14 i \\
2,52 & 4,01 & -0,01 \\
2,19+1,28 i & -0,35-1,07 i & 2,23+2,14 i
\end{array}\right]
\end{aligned}
$$

Thus, according to (11) for $\mathrm{D}^{(1)}$ we get the following results:

$$
\mathbf{D}^{(1)}=\left[\begin{array}{ccc}
-0,82-0,38 i & 0,26-0,31 i & 0,05+0,07 i \\
0,10+0,39 i & 0,82 & -0,10+0,39 i \\
0,05-0,07 i & -0,26-0,31 i & -0,82+0,38 i
\end{array}\right]
$$

The obtained values of the elements of the matrix $D^{(1)}$ coincide with those calculated by formula (9). 

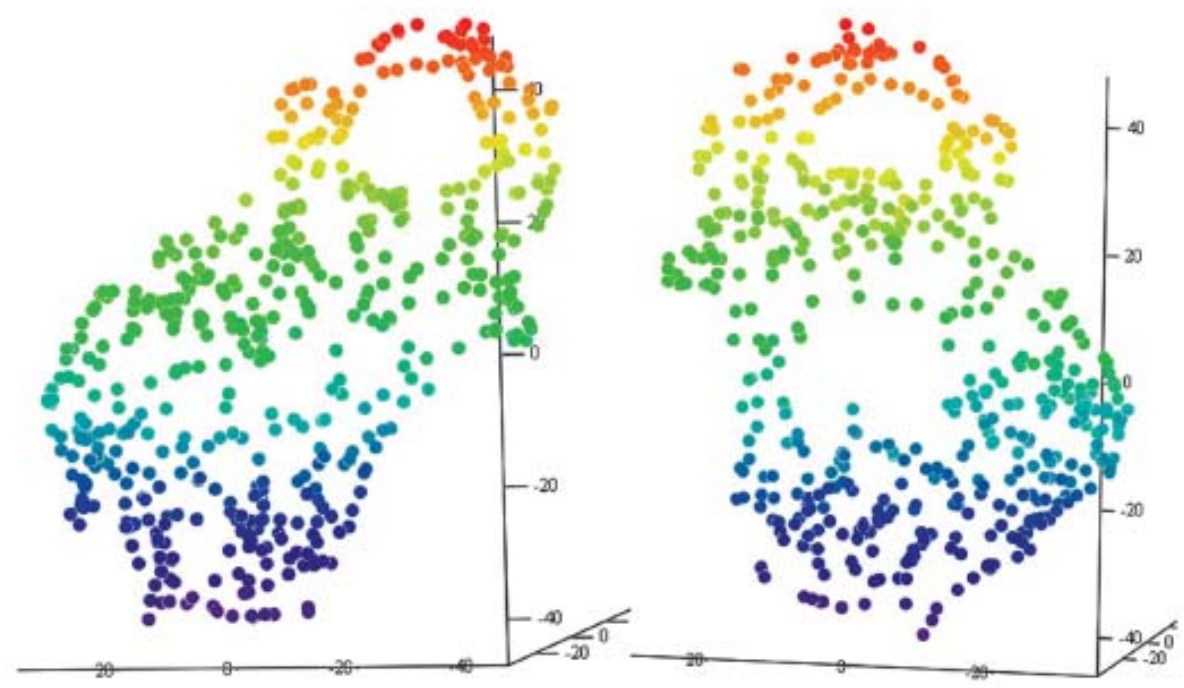

Figure 5: Examplesofpointfields, definingthereference (a) androtated (b) objects

\section{CONCLUSION}

Thus, the paper offers a comprehensive approach to processing three-dimensional images within the framework of solving a particular problem of estimating rotation parameters of three-dimensional objects whose surface is given by random references.

The authors developed a technique of parametrization of three-dimensional images, which makes it possible to process images of complex three-dimensional objects.

The paper shows the method for calculating the expansion coefficients in the basis of spherical harmonics for images of three-dimensional point cloud objects.

We propose an approach that provides the possibility of estimating the rotation parameters of three-dimensional objects from the values of the expansion coefficients in the basis of spherical harmonics.

The proposed approaches do not require to number the marks and make it possible to process objects specified by point clouds with a different number of references. Furthermore, these approaches are less complex, feasible for parallelization of computational operations, and do not involve iterative procedures, while ensuring uniqueness of solution.

\section{ACKNOWLEDGEMENT}

The work is executed at financial support of the Ministry of education and science of Russian Federation, project RFMEFI577170254 "System intraoperative navigation technology to support augmented reality-based virtual 3D models of organs obtained from the results of CT diagnostics, minimally invasive surgeries".

\section{REFERENCES}

1. Reich A M., \& Heipke C., (2014). Global approach for image orientation using lie algebraic rotation averageing and convex 10 minimisation. U: The International Archives of the Photogrammetry, Remote
Sensing and Spatial Information Sciences, 2014, Zurich, Switzerland. 265-272.

2. Jiang J., (2014). Estimation on Three-dimensional Motion Parameters in Binocular Vision of Robot Tracking and Control System. The Open Automation and Control Systems Journal, 6, 1498-1504.

3. Liwicki S., Pham M-T., Zafeiriou S., Pantic M., \& Stenger B., (2014). Full-Angle Quaternions for Robustly Matching Vectors of 3D Rotations. U: IEEE Conference on Computer Vision and Pattern Recognition, CVPR, 2014, Columbus, Ohio, USA. 105-112. doi:10.1109/CVPR.2014.21

4. Zhang J., Lv L., Shi X., Guo F., Zhang Y., \& Li H., (2013). Hough transform-based approach for estimating 3D rotation angles of vertebrae from biplanar radiographs using GPU-acceleration. International Journal of Imaging Systems and Technology, 23, 272-279.

5. Fredriksson J., (2016). Robust rotation and translation estimation in structure from motion. $U$ Mathematics Centre for Mathematical Sciences. Lund: Lund University.

6. Larsson V., Fredriksson J., Toft C., \& Kahl F., (2016). Outlier Rejection for Absolute Pose Estimation with Known Orientation. U: British Machine Vision Conference (BMVC), 2016, York, UK.

7. Khafizov D.G., (2012). Solution to the problem of ambiguity at combination of spatial point images by using the principal component analysis. Herald of Computer and Information Technologies, 7, 11-15.

8. Gonzalez-Barbosa J.J., Rico-Espino J.G., \& et al., . Accurate 3D Reconstruction using a Turntable-based and Telecentric Vision. AUTOMATIKA, 56(4), 508-521.

9. Li R., , Chen Y., Zhou C., \& Zhang L., (2015). Three-dimensional pose estimation model for object with complex surface. Advances in Mechanical Engineering, 1-12. doi:10.1177/168781401456848 
1. Rzazewska K., \& Luckner M., (2014). 3D model reconstruction and evaluation using a collection of points extracted from the series of photographs. U: the 2014 Federated Conference on Computer Science and Information Systems, 2014, Warsaw, Poland. 669-677.

2. Sakai K. \& Yasumura Y., (2018). Three-dimensional shape reconstruction from a single image based on feature learning. U: International Workshop on Advanced Image Technology 2018 (IWAIT 2018), 2018, Chiang Mai, Thailand.

3. Valgma L., (2016). 3D reconstruction using Kinect v2 camera. Tartu: University of Tartu.

4. Lawin F. J., (2015). Depth data processing and 3D reconstruction using the Kinect v2. Linkoping University.

5. Buriy A.S., \& Mikhailov S.N., (1994). Methods for identifying astro marks to navigate the spacecraft from images of the starry sky. Foreign Radio Electronics, 7-8, 44-52.

6. Furman Ya.A., Egoshina I.L., , \& Eruslanov R.V., - Matching Angular and Vector Descriptions of Three-Dimensional Group Point Objects. Avtometriya, 48(6), 3-17.

7. Egoshina I.L., (2013). Methods, models and algorithms for processing group point objects under conditions of a priori uncertainty of angular parameters.

8. Furman Ya.A., Eruslanov R.V., \& Egoshina I.L., (2013). Iterative algorithm for angular matching of group point objects with a priori uncertainty of parameters. Pattern Recognition and Image Analysis, 23(3), 381-388.

9. Furman Ya.A., \& Eruslanov R.V., (2011). Contour analysis of the task of the coordinate restoration of $3 \mathrm{~d}$ object points by the series images of its shades. Nonlinear World, 8, 482-486.

10. Anisimov B.V., (1993). Recognition and digital image processing. Moscow: Visshaya shkola.

11. Ballard, D. (1981). Generalizing the Hough transform to detect arbitrary shapes. Pattern Recognition, 13(2), 111-122.

12. Khoshelham, K. (2007). Extending general Hough transform to detect 3D object in laser range data. U: ISPRS Workshop on Laser Scanning 2007 and SilviLaser 2007, 2007, Espoo, Finland. 206-211.
13. Oliver, J.W. (2011). Demisting the Hough Tnsform for 3D Shape Recognition and registration. U: British Machine Vision Conference 2011, 2011, Dundee, Scotland. 1-11.

14. Pham, M., Woodford, O.J., Perbet, F., Maki, A., Stenger, B., \& Cipolla, R. (2011). A new distance for scale-invariant $3 \mathrm{D}$ shape recognition and registration. U: 2011 International Conference on Computer Vision, 2011, Barcelona, Spain. 145-152.

15. Chen S., \& Jiang H., (2011). Accelerating the hough transform with CUDA on graphics processing units. U: International Conference on Parallel and Distributed Processing Techniques and Applications (PDPTA), 2011, Las Vegas, USA.

16. Rozhentsov, A.A., Baev, A.A.,\& Naumov A.S., (2010). Estimation of Parameters and Recognition of Images of Three-Dimensional Objects with Disordered Samples. Avtometriya, 46, 57-69.

17. Huang H., Shen L., Zhang R., Makedon F., Hettleman B.,\& Pearlman J., (2005). Surface alignment of 3D spherical harmonic models: application to cardiac MRI analysis. Med Image Comput Comput Assist Interv, 8(1), 67-74.

18. Ritchie D., \& Kemp G., (1999). Fast Computation, Rotation and Comparison of Low Resolution Spherical Harmonic Molecular Surfaces. Journal of Computational Chemistry, 20(4), 383-395.

19. Buhmann M.D., (2004). Radial Basis Functions: Theory and Implementations. Cambridge: Cambridge University.

20. Titov V.S., Rozhentsov A.A., \& Khafizov R.G., (2014). Modern approaches to representation and processing of three-dimensional object images. Vestnik of Mari State Technical University. Series «Radio Engineering and Infocommunication Systems», 2, 44-54.

21. Brechbühler Ch., Gerig G., \& Kübler O., (1995). Parametrization of Closed Surfaces for 3-D Shape Description. Computer Vision and Image Understanding, 61(2), 154-170.

22. Tikhonov A.N., \& Arsent'ev V.Ya., (1979). Methods for solving ill-posed problems. Moscow: Nauka.

Paper submitted: 05.07.2018.

Paper accepted: 22.11.2018.

This is an open access article distributed under the CC BY-NC-ND 4.0 terms and conditions. 